\title{
A condição periodontal materna e 0 nascimento de prematuro de baixo peso: estudo caso-controle
}

\author{
Maternal periodontal status and preterm low birth weight: a case-control study
}

Fernanda Ferreira Lopes ${ }^{1}$, Liana Linhares Lima ${ }^{2}$, Maria Carmem de Albuquerque Rodrigues ${ }^{2}$, Maria Carmen Fontoura Nogueira da Cruz ${ }^{1}$, Ana Emília Figueiredo de Oliveira' ${ }^{1}$, Cláudia Maria Coelho Alves ${ }^{1}$

\section{RESUMO}

Objetivos: verificar as condições periodontais e necessidade de tratamento fornecidas pelo Registro Periodontal Simplificado (PSR) em puérperas, com o intuito de contribuir ao esclarecimento da relação entre doença periodontal e nascimento de recém-nascidos prematuros de baixo peso. Métodos: foi empregado o PSR em amostra de 40 puérperas, divididas em: grupo 1 (teste), composto pelas mães de recém-nascidos prematuros com peso inferior a $2.500 \mathrm{~g}(\mathrm{n}=20)$, e grupo 2 (controle), formado por mães de recém-nascidos a termo com peso igual ou superior a $2.500 \mathrm{~g}(\mathrm{n}=20)$. Os dados coletados foram analisados por meio de estatística descritiva, sendo os resultados do PSR submetidos ao tratamento estatístico para verificar se existem diferenças na condição periodontal e necessidade de tratamento entre as puérperas, sendo empregado o teste de Kolmogorov-Smirnov, em nível de significância de 5\%. Resultados: a presença de bolsa periodontal de 3,5 a 5,5 mm foi o achado mais comum entre as puérperas de recém-nascidos de baixo peso ( $39,17 \%$ dos sextantes), ao passo que a presença de sangramento à sondagem e ausência de bolsa periodontal foram os achados mais freqüentes entre as puérperas de recém-nascidos com peso normal (37,50\% dos sextantes), havendo diferença significativa na condição periodontal das puérperas $(\mathrm{p}=0,0494)$. Quanto à necessidade de tratamento, não houve diferença significativa entre os grupos estudados $(\mathrm{p} \geq 0,05)$. Conclusões: as puérperas de recém-nascidos prematuros com baixo peso apresentaram piores condições periodontais, sugerindo que a infecção periodontal pode estar relacionada ao nascimento de recém-nascidos prematuros de baixo peso.

PALAVRAS-CHAVE: Recém-nascido de baixo peso; Periodontia; Prematuro

\section{ABSTRACT}

Purpose: to evaluate periodontal conditions and need for treatment supplied by Periodontal Screening and Recording (PSR) in mothers, in order to clarify the relationship between periodontal disease and low birth weight premature newborns. Methods: PSR was used in a sample of 40 mothers, divided into: test group, consisting of mothers of premature newborns with weight less than $2,500 \mathrm{~g}(\mathrm{n}=20)$, and the control group, consisting of mothers of term newborns with a weight equal to or over 2,500 $\mathrm{g}(\mathrm{n}=20)$. The collected data were analyzed by descriptive statistics, and the results of PSR were submitted to statistical analysis in order to verify differences in periodontal condition and need for treatment of the mothers, using the Kolmogorov-Smirnov test, with a significance level of 5\%. Results: the presence of periodontal pocket of 3.5 to $5.5 \mathrm{~mm}$ was the most common finding among the mothers of newborns with low weight (39.2\% of the cases), while the presence of bleeding at probing and absence of a periodontal pocket were the most frequent findings among the mothers of newborns with normal weight (37.5\% of the cases). There was a statistically significant difference between periodontal conditions of the mothers of the groups ( $\mathrm{p}=0.0494)$, but in relation to the need for treatment, there was no significant difference between the studied groups $(\mathrm{p} \geq 0.05$ ). Conclusions: the mothers of preterm newborns with low weight presented worse periodontal conditions, suggesting that periodontal infection may be related to preterm low birth weight newborns.

KEYWORDS: Infant low birth weight; Periodontics; Infant, premature

Trabalho realizado no departamento de Odontologia da Universidade Federal do Maranhão - UFMA - São Luis (MA) - Brasil.

1 Docente do Curso de Odontologia da Universidade Federal do Maranhão - UFMA - São Luis (MA) - Brasil.

2 Cirurgiã-Dentista da Universidade Federal do Maranhão

Correspondência: Fernanda Ferreira Lopes

Endereço: Rua das Jaqueiras Qd. 57 Casa 19 - Bairro Renascença I - 65075-220 - São Luís - MA - Telefone (98) 3227-1318 - residencial 3217-8576 - Depto.Odonto II/UFMA - Fax: (98) 3235-9712 - e-mail: fernanda.f.lopes@gmail.com

Recebido em: 25/4/2005 
Introdução

Os recém-nascidos prematuros e de baixo peso (PMBP) representam, aproximadamente, 10\% de todos os nascimentos nos EUA, gerando custo anual que excede cinco bilhões de dólares. Embora os recém-nascidos PMBP sejam normais ao exame neurológico inicial, o espectro de deficiências neurológicas variam em diversos graus de anormalidades neuromotoras ${ }^{2}$, fazendo com que a prematuridade constitue problema perinatal mais sério em nossos dias, pois durante o período neonatal, os recém-nascidos PMBP apresentam taxa de morbidade 40 vezes mais elevada que os recém-nascidos a termo ${ }^{3}$.

É considerada gestação a termo aquela compreendida entre 37 semanas completas e menos de 42 semanas completas (259 a 293 dias completos), sendo os recém-nascidos de baixo peso aqueles que pesam menos de $2.500 \mathrm{~g}$ ao nascimento ${ }^{4}$. No entanto, as infecções maternas podem levar à presença de produtos bacterianos como lipopolissacarídeos ou endotoxinas provenientes de bactérias gram-negativas que estimulam a produção de citocinas, incluindo interleucina 1 (IL1), fator de necrose tumoral (TNF) e interleucina 6 (IL-6), e aumentam a produção de prostaglandinas, levando assim ao parto prematuro ${ }^{1,5}$.

As doenças periodontais são essencialmente infeciosas, baseadas na presença de determinadas espécies bacterianas, principalmente gram-negativas anaeróbicas, semelhante ao processo infecioso materno ${ }^{5}$. O objetivo final da terapia periodontal é a remoção dos patógenos causadores da doença e que, concomitantemente, haja recolonização por microrganismos compativeis com a saúde ${ }^{6,7}$.

O papel da infecção periodontal como possível fator de risco para o PMBP parece envolver a translocação de produtos bacterianos, tais como lipopolissacarídeos (LPS) ou mediadores inflamatórios (IL-1, IL-6, TNF- $\alpha$ e PGE $_{2}$ ), ao invés da passagem bacteriana, ou seja, translocação da bactéria por si só.

A associação significativa entre a gravidade da doença periodontal e recém-nascidos de baixo peso sugere a possibilidade de que a doença periodontal na gravidez seja fator de risco para o nascimento de recém-nascidos com baixo peso ${ }^{9,10}$, tendo sido detectado que a doença periodontal infecciosa parece ter relação direta com o nascimento de PMPB ${ }^{11}$. Todavia, alguns trabalhos mostraram não haver associação significativa entre a doença periodontal materna e o parto prematuro de recém-nascidos de baixo peso, ratificando que a doença periodontal materna não representa um fator de risco para o nascimento de $\mathrm{PMBP}^{12,13}$. As$\mathrm{sim}$, ainda existe a necessidade de mais estudos para esclarecer essa possivel relação, como evidenciado pelos dados microbiológicos da placa bacteriana subgengival ${ }^{14}$.

Diante da importância de conhecermos os fatores de risco associados ao parto prematuro e em face das discussões científicas sobre a possível associação entre a infecção periodontal e o nascimento de prematuros com baixo peso, tornamse imprescindiveis estudos abordando esses aspectos. Além do conflito de resultados apresentados na literatura e a sugestão de maior exploração do tema por trabalhos recentes ${ }^{14,15}$, o presente trabalho teve por objetivo verificar as condições periodontais e necessidade de tratamento fornecidas pelo Registro Periodontal Simplificado (PSR) em puérperas de recém-nascidos de baixo peso, por meio de estudo clínico caso-controle, com o intuito de contribuir ao esclarecimento da relação entre doença periodontal e nascimento de recém-nascidos prematuros de baixo peso.

\section{Métodos}

A presente pesquisa consiste de estudo observacional do tipo caso-controle, no qual foram examinadas 40 puérperas. Para inclusão na amostra, o parto de todas as mães teria que ter sido realizado no Hospital Universitário Materno Infantil da Universidade Federal do Maranhão (HUUFMA), sendo considerado critério de exclusão quando no dia do exame bucal, o parto havia sido realizado em periodo superior a três dias. A amostra selecionada foi dividida em dois grupos: grupo teste (Gpmbp), composto pelas mães que tiveram recém-nascidos prematuros com peso inferior a $2.500 \mathrm{~g}(\mathrm{n}=20)$ e o grupo controle (Gbt), formado por puérperas cujos recém-nascidos a termo tinham peso igual ou superior a $2.500 \mathrm{~g}(\mathrm{n}=20)$.

A amostra era formada por mulheres com idade entre 15 a 28 anos, sendo que as mães de recém-nascidos prematuros de baixo peso (Gpmbp) apresentaram idade variando de 15 a 27 anos (média de 21, 4 anos) e nas com recém-nascidos a termo (Gbt), a idade variou de 15 a 28 anos (média de 20, 9 anos) .

Durante a seleção da amostra foram investigados os possiveis fatores de risco para o nascimento de recém-nascidos PMBP, tais como idade das gestantes, consumo de fumo e álcool durante a gravidez, primiparidade e infecções. Estes dados foram coletados por meio de entrevista interpessoal. Além disso, os prontuários de cada 
paciente foram analisados visando a confirmação dos dados fornecidos pelas mães acrescidos das informações pertinentes ao recém-nascido. As características das puérperas dos grupos teste e controle apresentaram certa similaridade, quanto a idade, primiparidade, cuidados pré-natais e hipertensão (Tabela 1).

Tabela 1 - Características das puérperas com recém-nascidos de baixo peso (Gpmbp) comparadas com as dos recém-nascidos com peso normal (Gbt), quanto às variáveis estudadas.

\begin{tabular}{lcc}
\hline Características & $\begin{array}{c}\text { Gpmbp } \\
(\mathbf{n}=\mathbf{2 0})\end{array}$ & $\begin{array}{c}\text { Gbt } \\
(\mathbf{n}=\mathbf{2 0})\end{array}$ \\
\hline Idade & $21,45 \pm 3,15$ anos & $20,85 \pm 4,10$ anos \\
Peso do recém-nascido & $1.895,5 \pm 320,6 \mathrm{~g}$ & $3.356,9 \pm 416,73 \mathrm{~g}$ \\
Tempo de gestação & $34,20 \pm 1,24$ semanas & $39,20 \pm 1,51$ semanas \\
Primíparas & 10 & 10 \\
Existência de pré-natal & 18 & 19 \\
Infecção do trato genito-urinário & 5 & 10 \\
Diabetes & 0 & 0 \\
Fumante & 4 & 0 \\
Uso de álcool & 3 & 0 \\
Hipertensão & 5 & 7 \\
\hline
\end{tabular}

Para o exame bucal, foi escolhido o PSR com indice de estudo, por coletar informações concernentes à condição periodontal e necessidade de tratamento. Para tanto foi utilizada a sonda WHO621, que possui uma ponta esférica de $0,5 \mathrm{~mm}$ de diâmetro e uma área codificada preta de 3,5 a 5,5 $\mathrm{mm}$. Por meio dessa sonda periodontal, todos os dentes de cada paciente foram examinados em seis pontos (mésio-vestibular, médio-vestibular, disto-vestibular, mésio-lingual, médio-lingual e disto-lingual). Os dentes foram classificados de acordo com as seguintes categorias ${ }^{16,17}$ : código 0 : faixa colorida totalmente visivel, sem sangramento à sondagem, ausência de cálculos e excessos de restaurações; código 1: faixa colorida totalmente visivel, embora com presença de sangramento à sondagem; sem cálculos e excessos de margens das restaurações; código 2: faixa colorida totalmente visivel, porém presença de sangramento à sondagem, presença de cálculos supra e/ou subgengivais; código 3: faixa colorida parcialmente visivel, ou seja, presença de bolsa periodontal de 3,5 a 5,5 mm; código 4: faixa colorida não visivel, ou seja, totalmente no interior da bolsa (presença de bolsa periodontal acima de $5,5 \mathrm{~mm}$ ).

Para as pacientes classificadas com o código 0 , apenas há necessidade de se manter a paciente sob medidas preventivas adequadas; código 1 , as necessidades de tratamento consistem em eliminação da placa supragengival pelo profissional e instrução de higiene bucal; código 2 , neces- sidade de raspagem e polimento das superficies dentais, além de medidas preventivas de higiene bucal; código 3 , corresponde à necessidade de tratamento periodontal especializado do sextante; código 4, necessidade de minucioso exame periodontal completo de toda boca, além de tratamento periodontal especializado e complexo.

Seguindo a metodologia do PSR, considerou-se para registro de dados o maior escore em cada grupo de dentes denominados de sextantes (lados direito e esquerdo e bateria anterior da maxila e mandíbula). Na presença de um único dente ou ausência de todos em cada sextante aplicava-se o código X.

Os dados coletados foram analisados por meio de estatística descritiva, sendo os resultados do PSR submetidos ao tratamento estatístico para verificar se existiam diferenças na condição periodontal e necessidade de tratamento entre as puérperas, sendo empregado o teste de KolmogorovSmirnov, em nível de significância de $5 \%$.

Este trabalho foi aprovado pelo Comitê de Ética do Hospital Universitário Presidente Dutra (Processo 33104-00404/2002), conforme a Resolução no 196/ 1996 do Conselho Nacional de Saúde (CNS) do Ministério da Saúde, quanto ao consentimento livre e esclarecido dos indivíduos para a participação da pesquisa.

\section{Resultados}

Observou-se que a puérperas com PMPB (Gpmbp) apresentaram piores condições periodontais que as mães dos recém-nascidos a termo (Gbt), com maior prevalência dos escores 3 e 4 medidos pelo PSR nos sextantes das puérperas do Gpmbp. A Tabela 2 mostra que o código 3 foi o mais comum entre as puérperas de recém-nascidos de baixo peso $(39,2 \%$ dos sextantes), ao passo que o código 1 foi o mais freqüente nas puérperas de recém-nascidos com peso normal $(37,5 \%$ dos sextantes), havendo diferença significativa na distribuição entre os grupos avaliados (teste de Kolmogorov-Smirnov; $\alpha=0,05 ; p=0,0494)$.

Tabela 2 - Distribuição percentual dos códigos do PSR por casos, em puérperas com recém-nascidos de baixo peso (Gpmbp) comparadas com as dos recém-nascidos com peso normal (Gbt).

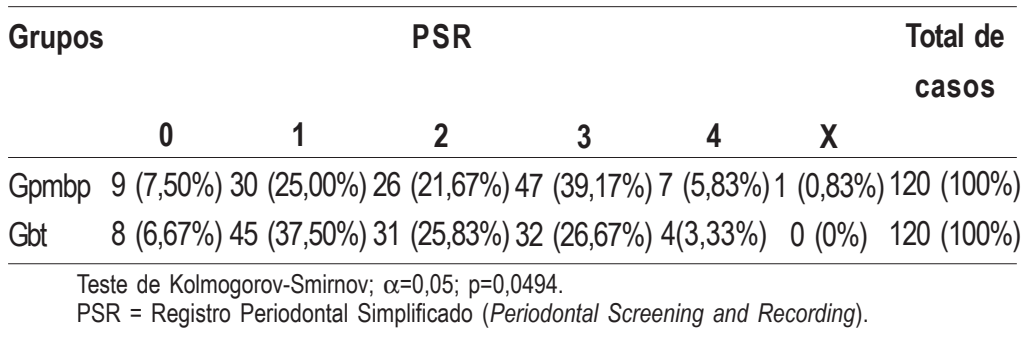


Todas as puérperas apresentaram alguma necessidade de tratamento (NT), seja de forma mais simples (códigos 1 e 2) ou especializada (códigos 3 e 4) para ambos os grupos teste e controle. Observou-se que as necessidades de tratamento especializado (NT3) e especializado complexo (NT4) estavam presentes em 50 e $30 \%$ das puérperas do Gpmbp e em 55 e 15\% das puérperas do Gbt, respectivamente, não havendo diferença significativa quanto à NT entre os grupos estudados (teste de Kolmogorov-Smirnov; $\alpha=0,05 ; p \geq 0,05)$. A Tabela 3 mostra a distribuição percentual das puérperas de recém-nascidos de baixo peso e de peso normal, segundo a necessidade de tratamento.

Tabela 3 - Distribuição das puérperas de recém-nascidos de baixo peso (Gpmbp) e de recém-nascidos de peso normal (Gbt), segundo necessidade de tratamento periodontal (NT) pelo PSR.

\begin{tabular}{lrlllll}
\hline Puérperas & \multicolumn{3}{c}{ PSR } & & & Total \\
& NT0 & NT1 & NT2 & NT3 & NT4 & \\
\hline
\end{tabular}

Gpmbp $0(0 \%) 1(5 \%) 3(15 \%) 10(50 \%) 6(30 \%) 20(100 \%)$

Gbt $\quad 0(0 \%) 0(0 \%) 6(30 \%) 11(55 \%) 3(15 \%) 20(100 \%)$

Teste de Kolmogorov-Smirnov; $\alpha=0,05 ; p \geq 0,05$.

PSR = Registro Periodontal Simplificado (Periodontal Screening and Recording).

\section{Discussão}

Por ser considerado método simples, sensível e objetivo para o diagnóstico da doença periodontal ${ }^{17}$, o PSR foi o índice escolhido na metodologia do presente estudo. Além dessa particularidade, o PSR demonstra ser eficiente para monitorar as pacientes, tanto aquelas com história de doença periodontal, como aquelas que necessitam de tratamento ${ }^{16}$.

O presente trabalho chama atenção para as diferenças significativas nas condições periodontais das puérperas e sua possível relação com o nascimento de recém-nascidos de baixo peso. As puérpuras de recém-nascidos PMBP apresentaram piores condições periodontais, quando o escore 3 (presença de bolsa periodontal entre 3,5 a $5,5 \mathrm{~mm}$ ) foi o código mais freqüente, ao passo que as puérperas de recém-nascidos com peso normal apresentaram o escore 1 (presença de sangramento gengival com ausência de bolsa periodontal) como o código mais prevalente.

Os achados dessa pesquisa são similares ao estudo de Mokeem et al. ${ }^{18}$, que encontraram elevada prevalência de doença periodontal entre as puérperas de recém-nascidos PMBP, ratificando a correlação entre parto prematuro de recém-nascidos de baixo peso e a doença periodontal.
A infecção é considerada uma das causas mais importantes para o parto de $\mathrm{PMBP}^{3}$, no entanto, convém ressaltar que, no presente estudo, as puérperas do Gbt apresentaram maior freqüência de infecção no trato gênito-uninário que as do Gpmbp. Tal resultado corrobora a hipótese de que a infecção local não é única causa dessa condição ${ }^{3}$, pois a maior prevalência de sextantes com infecção periodontal das puérperas do Gpmbp (PSR 3 e 4), observada nessa pesquisa, ratifica o papel da doença periodontal como a possivel infecção associada ao parto prematuro de recém-nascidos de baixo peso ${ }^{1,12}$.

Os presentes resultados encontram fundamentação no experimento realizado por Collins et al. ${ }^{19}$, que detectaram diminuição em cerca de $20 \%$ do peso dos filhotes de hamsters, cujas mães foram sensibilizadas com patógenos periodontais. São compativeis ainda com os dados de Offenbacher et al. ${ }^{11}$ ao concluirem que doença periodontal foi responsável por $18,2 \%$ dos casos de nascimento de recém-nascidos PMBP. Esses autores ressaltam que as infecções periodontais servem de reservatório para organismos gram-negativos anaeróbios, LPS, endotoxinas e mediadores inflamatórios incluindo $\mathrm{PGE}_{2}$ e TNF- $\alpha$, que podem representar uma ameaça potencial para a unidade feto-placentária.

Porém, o presente relato diverge do trabalho de Teng et al. ${ }^{12}$, ao mencionarem que a doença periodontal materna não representa fator de risco para o nascimento de recém-nascidos de baixo peso, hipótese essa alicerçada pelos resultados de Davenport et al. ${ }^{13}$, que não encontraram associação significativa entre doença periodontal materna e PMBP, apesar de terem observado decréscimo no peso dos recém-nascidos com o aumento da perda de inserção periodontal das mães.

Ratifica-se que a relação causal entre doença periodontal e o peso dos neonatos ainda não está esclarecida ${ }^{14,15,20}$. Todavia, convém ressaltar que no presente trabalho as puérperas com recémnascidos prematuros e de baixo peso apresentaram piores condições periodontais do que as que tiverem recém nascidos a termo com peso normal, semelhante a diversos estudos presentes na literatura ${ }^{9,11,14,18}$.

Não foram investigados outros fatores de risco para o trabalho de parto prematuro, infeccioso ou não. No entanto, os resultados obtidos indicam que todas as gestantes devem procurar assistência odontológica, pois a terapia periodontal reduziu significativamente as taxas de partos prematuros de recém-nascidos de baixo peso em mulheres com doença periodontal ${ }^{20}$. Concluiu-se que a doença periodontal é condição que pode ser preve- 
nida e tratada, e que também se faz recomendar a inclusão de programas preventivos de saúde oral para as gestantes durante o pré-natal ${ }^{21-22}$.

Com base nos resultados desta pesquisa, pode-se concluir que as puérperas de recém-nascidos prematuros com baixo peso apresentaram significativamente piores condições periodontais, sugerindo que a infecção periodontal pode estar relacionada ao nascimento de prematuros de baixo peso.

\section{Referências}

1. Offenbacher S, Jared HL, O'Reilly PG, Wells SR, Salvi GE, Lawrence HP, et al. Potential pathogenic mechanism of periodontitis associated pregnancy complications. Ann Periodontal. 1998;3(1):233-50.

2. Hack M, Caron B, Rivers A, Fanaroff AA. The very low birth weight infant: the broader spectrum of morbidity during infancy and early childhood. J Dev Behav Pediatr. 1983;4(4):243-9.

3. McGaw T. Periodontal disease and preterm delivery of low-birth-weight infants. J Can Dent Assoc. 2002;68(3):165-9.

4. Rezende J, Montenegro CAB. Obstetrícia fundamental. 9a ed. Rio de Janeiro: Guanabara Koogan; 2002.

5. Carta G, Persia G, Falciglia K, Iovenitti P. Periodontal disease and poor obstetrical outcome. Clin Exp Obstet Gynecol. 2004;31(1):47-9.

6. Lotufo R, Pannuti C. Recursos para o diagnóstico microbiológico em periodontia. In: Oppermann R, Rösing C, editores. Periodontia: ciência e clínica. São Paulo: Artes Médicas; 2001. p. 31-8.

7. Feres M, Gonçalves C. O impacto do diagnóstico microbiológico na terapêutica periodontal. In: Oppermann R, Rösing C, editores. Periodontia: ciência e clínica. São Paulo: Artes Médicas; 2001. p. 39-56.

8. Gibbs RS, Romero R, Hillier SL, Eschenbach DA, Sweet RL. A review of premature birth and subclinical infections. Am J Obstest Gynecol. 1992;166(5):1515-28.

9. Louro PM, Fiori HH, Louro Filho P, Steibel J, Fiori RM. Doença periodontal na gravidez e baixo peso ao nascer. J Pediatr (Rio de J). 2001;77(1):23-8.

10. Marin, C, Segura-Egea JJ, Martinez-Sahuquillo A, Bullón P. Correlation between infant birth weight and mother's periodontal status. J Clin Periodontol. 2005;32(3):299-304.
11. Offenbacher S, Katz V, Fertik G, Collins J, Boyd D, Maynor G, et al. Periodontal infection as possible risk factor for preterm low birth weight. J Periodontol. 1996;67(10 Suppl):1103-13.

12. Teng YT, Taylor GW, Scannapieco F, Kinane DF, Curtis $\mathrm{M}$, Beck JD, et al. Periodontal health and systemic disorders. J Can Dent Assoc. 2002;68(3):188-92.

13. Davenport ES, Williams CE, Sterne JA, Murad S, Sivapathasundram V, Curtis MA. Maternal periodontal disease and preterm low birth weigth: case-control study. J Dent Res. 2002;81(5):313-8.

14. Buduneli, N, Baylas H, Buduneli E, Türkoglu O, Köse T, Dahlen G. Periodontal infections and pre-term low birth weight: a case-control study. J Clin Periodontol. 2005;32(2):174-81.

15. Scannapieco FA, Bush RB, Paju S. Periodontal disease as a risk factor for adverse pregnancy outcomes. A systemic review. Ann Periodontol. 2003;8(1):70-8.

16.Zenóbio E, Moreira MM, Santos FA, Toledo BEC. Registro periodontal simplificado (PSR). Rev CROMG. 1998;4(1):38-41.

17. Rossell FL, Montandon-Pompeu AAB, Valsecki Junior A. Registro periodontal simplificado em gestantes. Rev. Saúde Pública. 1999;33(2):152-62.

18. Mokeem SA, Molla GN, Al-Jewair TS. The prevalence and relationship between periodontal disease and pre-term low birth weight infants at King Khalid University Hospital in Riyadh, Saudi Arabia. J Contemp Dent Pract. 2004;5(2):40-56.

19. Collins JG, Windley HW 3rd, Arnold RR, Offenbacher S. Effects of Porphyromonas gingivalis infection on inflammatory mediator response and pregnancy outcome in hamsters. Infect Immun. 1994;62(10):4356-61.

20.Williams CE, Davenport ES, Sterne JA, Sivapathasundaram VV, Fearne JM, Curtis MA. Mechanisms of risk in preterm low-birthweight infants. Periodontol 2000. 2000;23(1):142-50.

21.Lopez NJ, Smith PC, Gutierrez J. Periodontal therapy may reduce the risk of preterm low birth weight in women with periodontal disease: a randomized controlled trial. J Periodontol. 2002;73(8):911-24.

22.Yeo BK, Lim LP, Paquette DW, Williams RC. Periodontal disease - the emergence of a risk for systemic conditions: pre-term low birth weight. Ann Acad Med Singapore. 2005;34(10):111-6. 